\title{
Author's Reply: Hemidystonia caused by frontal cortical infarction
}

\author{
Vladimir Miletić ${ }^{1}$
}

Received: 19 September 2015/ Accepted: 12 October 2015/Published online: 4 November 2015

(C) Belgian Neurological Society 2015

Sir,

We read with interest the comments of Prof. Garraux on our recent case report describing a 71-year-old woman presenting with right-sided hemidystonia following an ischemic stroke. The initial title of our paper was: "Hemidystonia caused by a frontoparietal cortical infarction". However, we now receive the comment that according to the published computed tomography axial images of the brain, the causal lesion is located in the postcentral gyrus of the left parietal lobe.

Indeed, we must agree that the lesion shown in image $1 \mathrm{~A}$ of the manuscript is located in the left postcentral gyrus of the parietal lobe.

\section{Compliance with ethical standards}

Conflict of interest The author declares that he has no conflict of interests.

Ethical approval All applicable international, national and institutional guidelines for the treatment of patient were followed.

Informed consent Written informed consent was obtained from the patient for publication of this case report and any accompanying images.
Vladimir Miletić

vladimir.miletic@gmail.com

1 Department of Neurology, Movement Disorders Centre, University Hospital Centre Zagreb, Kispaticeva 12, 10000 Zagreb, Croatia 